\title{
Adsorption of Spherical Polyelectrolyte Brushes: from Interactions to Surface Patterning
}

\author{
By Christoph Hanske ${ }^{1}$, Johann Erath ${ }^{1}$, Christin Kühr ${ }^{2}$, Martin Trebbin ${ }^{2}$, \\ Christian Schneider ${ }^{2,3}$, Alexander Wittemann ${ }^{4}$, and Andreas Fery ${ }^{1, *}$ \\ ${ }^{1}$ Physical Chemistry II, University of Bayreuth, 95440 Bayreuth, Germany \\ ${ }^{2}$ Physical Chemistry I, University of Bayreuth, 95440 Bayreuth, Germany \\ ${ }^{3}$ F-I2 Soft Matter and Functional Materials, Helmholtz-Zentrum Berlin, 14109 Berlin, Germany \\ ${ }^{4}$ Department of Chemistry, University of Constance, 78464 Konstanz, Germany
}

Dedicated to Matthias Ballauff on the occasion of his $60^{\text {th }}$ birthday

(Received April 30, 2012; accepted in revised form July 6, 2012)

(Published online August 6, 2012)

\section{Spherical Polyelectrolyte Brushes / Adsorption / Particles / Colloidal Probe / AFM / Microcontact Printing / Surface Patterning}

Adsorption of colloidal particles constitutes an attractive route to tailor the properties of surfaces. However, for efficient material design full control over the particle-substrate interactions is required. We investigate the interaction of spherical polyelectrolyte brushes (SPB) with charged substrates based on adsorption studies and atomic force spectroscopy. The brush layer grafted from the colloidal particles allows a precise adjustment of their adsorption behavior by varying the concentration of added salt. We find a pronounced selectivity between oppositely and like-charged surfaces for ionic strengths up to $10 \mathrm{mM}$. Near the transition from the osmotic to the salted brush regime at approximately $100 \mathrm{mM}$ attractive secondary interactions become dominant. In this regime SPB adsorb even to like-charged surfaces. To determine the adhesion energy of SPB on charged surfaces directly, we synthesize micrometer-sized SPB. These particles are used in colloidal probe AFM studies. Measurements on oppositely charged surfaces show high forces of adhesion for low ionic strengths that can be attributed to an entropy gain by counterion release. Transferring our observations to charge patterned substrates, we are able to direct the deposition of SPB into two-dimensional arrays. Considering that numerous chemical modifications have been reported for SPB, our studies could open exiting avenues for the production of functional materials with a hierarchical internal organization.

\section{Introduction}

The physisorption of components from solution constitutes a versatile and easily upscalable alternative to surface modifications based on covalent chemical coupling, since coupling by physisorption does not require specific chemical reactions to occur.

* Corresponding author. E-mail: andreas.fery@uni-bayreuth.de 
A prominent example for this approach is the layer-by-layer (LbL) deposition of oppositely charged polyelectrolytes [1-4]. Multilayers can be applied to large substrates by simple dip or spray coating and the non-covalent nature of the underlying interactions allows for a broad spectrum of molecular components [5-8]. Other examples are the physisorption of microgel-particles [9] which can be used as temperature-responsive cell substrates [10] or the adsorption of responsive block-copolymer micellar aggregates [11] for controlled release and cellular response.

Indeed often colloidal building blocks are integrated in such physisorptionlayers [12-16], since they carry functions (optical, electronic, catalytic, magnetic properties or responsiveness towards various stimuli), but also because the size of colloidal particles increases the adsorption energy as compared to single (macro-)molecules while still ensuring that interfacial interactions are dominant over inertia or other forces governing the macro-scale. The efficiency of these materials depends on both the physical properties of the colloids and their spatial arrangement on the substrate.

Modification of the colloidal building blocks by grafting polymer chains prior to physisorption is a promising strategy for optimizing these aspects inasmuch as the surface layer moderates the interaction with the substrate independent of the type of particles. Such an approach can be applied to any type of colloids including inorganic and polymer particles. If the packing of the chains is sufficiently dense, i.e. the lengths of the chains tethered with one end to the particle must be considerably larger than the distance between two neighboring chains, a polymer brush results [17]. Moreover, if the brush is made from polyelectrolytes, it adds electrosteric stabilization, stimuli-responsiveness and compatibility as well as adhesiveness or nonadhesiveness to particles that could exhibit special optical, mechanical or magnetic features, thus establishing multi-functional building blocks [18-21]. Because of the spherical geometry of the colloidal support, such particles are denoted as "spherical polyelectrolyte brushes" (SPB). The core-shell morphology of SPB is schematically depicted in Fig. 1A.

The properties of SPB in solution are widely determined by the confinement of the counterions of the polyelectrolyte chains. Approximately $95 \%$ of the counterions of the polyelectrolyte chains are trapped within the brush at low concentrations of added salt [22]. This creates a huge osmotic pressure resulting in a marked stretching of the polyelectrolytes $[23,24]$. The responsiveness of the SPB towards external stimuli such as the ionic strength and $\mathrm{pH}$ allows precise control over the spatial dimensions of the particles and their mutual interaction [25-28].

Functionalization of the core-shell colloids can be accomplished by loading the core with hydrophobic substances, including many drugs. Moreover, the surrounding brush layer may serve as a carrier for active nanostructures, namely for metal nanoparticles, enzymes and conductive polymers yielding functional colloids with tailored biological, catalytic or electronic properties [29-32]. This opens up avenues for multifunctional responsive mesoscopic building units that are stable against coagulation and can be easily handled [33].

Understanding the interaction of SPB with solid substrates is a prerequisite for their technological application in functional coatings. Studies with mica surfaces demonstrated distinct differences in the adsorption behavior of cationic and anionic SPB [34, 35]. While anionic SPB exhibited a high lateral mobility on the negative substrates and 

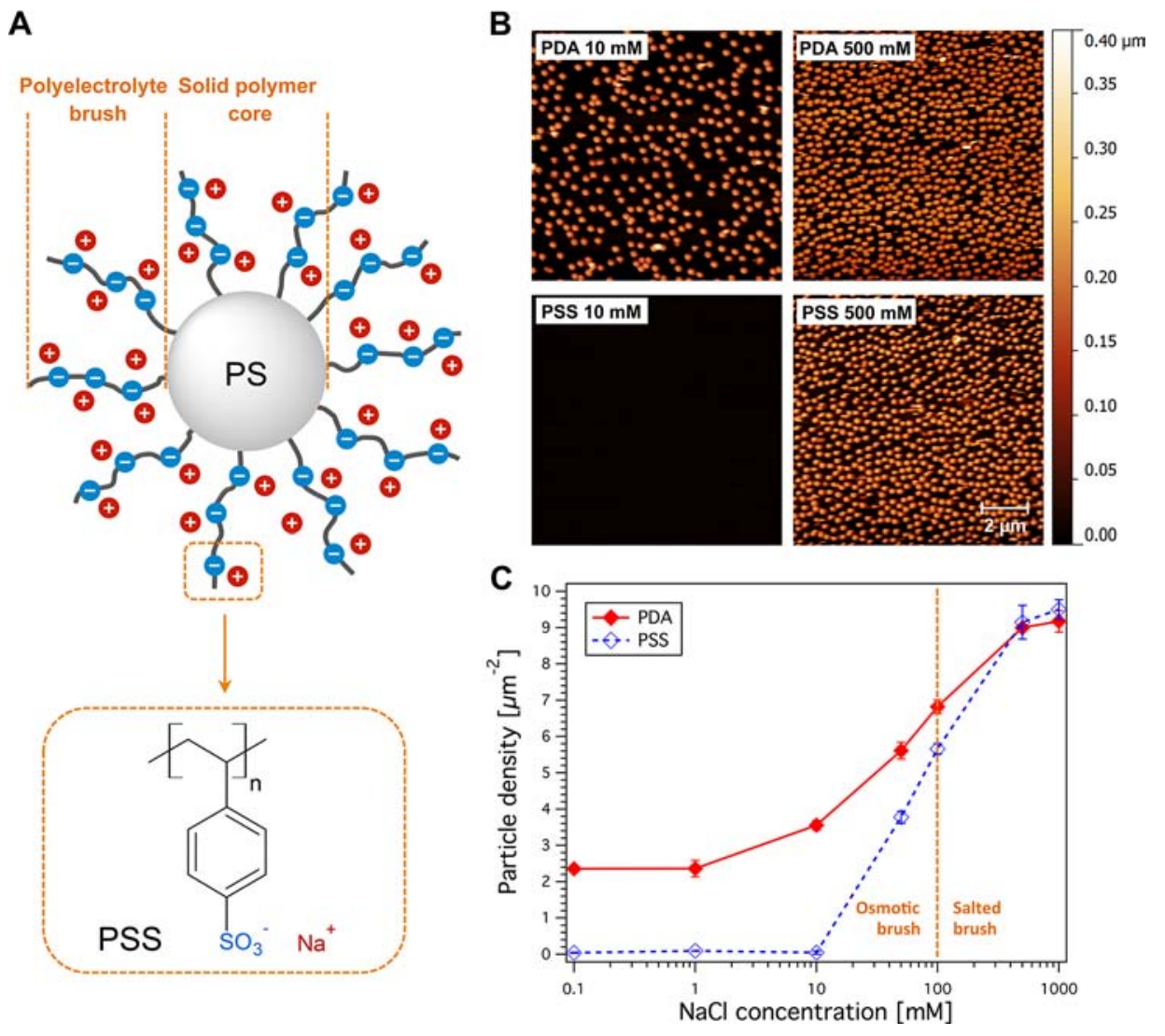

Fig. 1. Influence of substrate charge and ionic strength on SPB adsorption. For this study anionic SPB consisting of a PS core and attached PSS chains were used (A). Before drying, the films exhibit a liquidlike particle ordering indicating a random adsorption process (B). At $10 \mathrm{mM}$ of added salt the particles bind only to oppositely charged surfaces, whereas particle deposition at $500 \mathrm{mM}$ results in a high surface coverage on both types of substrates. The equilibrium particle density plotted against the ionic strength shows that the loss of substrate selectivity coincides with the transition from the osmotic to the salted brush regime near $100 \mathrm{mM}(\mathrm{C})$.

formed hexagonally packed arrangements during drying, cationic SPB were strongly affixed to the surface resulting in network-like structures. Recently, we investigated SPB adsorption onto polyelectrolyte multilayers focusing on the kinetics [36]. We found that after an initial diffusion-limited stage SPB adsorption slows down and finally ceases with the formation of a particle monolayer.

In this work we investigate the interaction of anionic SPB and polyelectrolyte multilayers consisting of polystyrene sulfonate (PSS) and poly(diallyldimethylammonium chloride) (PDA). In addition to adsorption studies covering a wide range of ionic strengths [36], we present a direct assessment of the force of adhesion via atomic force microscopy (AFM). We utilize the colloidal probe (CP) technique, in which force-distance curves are recorded with an AFM cantilever bearing a micrometer-sized 
spherical particle [37]. This method was developed independently by Butt and Ducker and allows a normalization of the measured forces over the contact area by the Derjaguin approximation [38,39]. Another advantage of CP-AFM is the possibility to use functionalized particles and measure the interaction between arbitrary surfaces including polyelectrolyte brushes [40-43]. Building upon the synthetic route to submicron SPB originally developed by Ballauff and co-workers [24,25], we attach PSS chains to micrometer-sized polystyrene particles. These SPB microparticles are then used to measure the interaction of SPB with charged surfaces. Finally, we demonstrate that under appropriate deposition conditions SPB can be arranged into well-defined arrays on charge patterned substrates. For this purpose we utilize microcontact printing, which is a prominent technique to facilitate surface patterning and guided adsorption [44-46].

\section{Experimental section}

\subsection{Materials}

Styrene was passed through a catechol inhibitor remover column before use. Irgacure 2959 was kindly supplied by Ciba Specialty Chemicals and transferred into the copolymerizable photoinitiator (HMEM) by a Schotten-Baumann reaction of Irgacure 2959 and methacrylic acid hydrochloride along the lines given in Ref. [24]. Purification was accomplished by column chromatography on silica gel. The purity of the product was verified through NMR spectroscopy (AC 250, Bruker). Deionized water obtained from a reverse osmosis water purification system (Millipore Academic A10) was used throughout the entire studies. All latexes were purified by exhaustive ultrafiltration against deionized water. The other chemicals and solvents were of analytical grade and were used as received.

\subsection{Nanoparticles synthesis}

The synthesis and characterization of submicron SPB following the approach of Ballauff and coworkers are described in Ref. [47]. Briefly, polystyrene cores bearing covalently anchored photoinitiator moieties were produced by soap-free emulsion polymerization in the presence of a UV sensitive comonomer. From the particle surfaces PSS chains were grafted by UV induced polymerization of sodium styrene sulfonate. The PS cores display a narrow size distribution with an average radius of $126 \pm$ $2 \mathrm{~nm}$ as measured by dynamic light scattering (DLS). The hydrodynamic thickness of the PSS brush is $74 \pm 3 \mathrm{~nm}$ in deionized water. The PSS chains were cleaved from the PS cores and analyzed by size exclusion chromatography. The molecular weight of the longest PSS chains that govern the spatial extension of the brush layer was determined as $67600 \pm 4950 \mathrm{~g} / \mathrm{mol}$, which corresponds to a contour length of $82 \pm 6 \mathrm{~nm}$ [47]. The polydispersity of the chains (weight averaged molecular weight by number averaged molecular weight) was calculated as 2.1 , which is a common value for polymers prepared by free radical polymerization. The chain grafting density is $0.03 \pm 0.01$ chains per $\mathrm{nm}^{2}$ [47]. 


\subsection{Microparticles synthesis}

PSS brushes were grafted from monodisperse cross-linked PS microparticles with a diameter of $4.8 \mu \mathrm{m}(\mathrm{SX}-500 \mathrm{H})$ which were kindly supplied from Soken Chemical \& Engineering Co.

The photoinitiator layer surrounding the microparticles was formed in a seeded growth polymerization. Briefly, $15 \mathrm{~g}$ of the microparticle powder was dissolved in $14 \mathrm{~g}$ ethanol yielding a homogeneous suspension after sonication for $2 \mathrm{~min} .143 \mathrm{~g}$ deionized water was added dropwise under continuous stirring followed by further sonication to minimize agglomeration. The PS seeds were swollen with $1.13 \mathrm{~g}$ styrene (injection rate $0.02 \mathrm{~g} / \mathrm{ml}$ ) and stirred for a period of $15 \mathrm{~h}$ at $130 \mathrm{rpm}$. The polymerization was performed at $70{ }^{\circ} \mathrm{C}$ under a nitrogen atmosphere and continuous stirring ( $\left.300 \mathrm{rpm}\right)$. To initiate the reaction, $0.284 \mathrm{~g}$ potassium persulfate was added. After $15 \mathrm{~min}, 2.098 \mathrm{~g}$ of a $69.6 \mathrm{wt} \%$ solution of the copolymerizable photoinitiator HMEM in acetone was injected into the suspension (rate $0.05 \mathrm{~g} / \mathrm{min}$ ). The reaction was allowed to proceed for $2 \mathrm{~h}$. The microparticles were isolated from the dispersant, redispersed in ethanol and stored in a $1: 1$ ethanol-water mixture for further use.

Grafting of the polyelectrolyte brushes was carried out in a closed reaction chamber containing a UV emitter with a focusable reflector (Hoenle UV Technology UV-F 400 F). An iron doped metal halogenide lamp with a power of $400 \mathrm{~W}$ was used in combination with a blue filter (transmission window: $320-450 \mathrm{~nm}$ ). $44 \mathrm{mg}$ of sodium styrene sulfonate was added to suspensions of $217 \mathrm{mg}$ photoinitiator-coated microparticles dispersed in $4.8 \mathrm{~g}$ of a $1: 1$ ethanol-water solution. The reaction mixtures were irradiated with UV for $30 \mathrm{~min}$ at r.t. under permanent stirring. Purification of the suspension was accomplished by exhaustive ultrafiltration against deionized water.

\subsection{Polyelectrolyte solutions}

All polyelectrolytes were used as aqueous solutions containing $1 \mathrm{~g} / 1$ and varying amounts of $\mathrm{NaCl}$ (99.88\%, Fisher Scientific). Multilayer coatings were produced with the following polyelectrolytes (Aldrich): PEI (poly(ethylene imine), $\mathrm{MW}=25000 \mathrm{~g} / \mathrm{mol}$ ), PSS (poly(sodium-4-styrene sulfonate), $\mathrm{MW}=70000 \mathrm{~g} / \mathrm{mol}$, $50 \mathrm{mM} \mathrm{NaCl}$ ), PDA (poly(diallyldimethylammonium chloride), $\mathrm{MW}=100000$ $200000 \mathrm{~g} / \mathrm{mol}, 50 \mathrm{mM} \mathrm{NaCl}$ ). For microcontact printing we used fluorescently labeled PDA-TRITC (Surflay, MW $=70000 \mathrm{~g} / \mathrm{mol}, 500 \mathrm{mM} \mathrm{NaCl}$ ).

\subsection{Substrate preparation}

Glass slides and silicon wafers were cut into pieces of $10 \mathrm{~mm} \times 25 \mathrm{~mm}$ and cleaned by the RCA method using analytical grade chemicals (2-propanol, $\mathrm{NH}_{3}, \mathrm{H}_{2} \mathrm{O}_{2}$ from VWR) [48]. Functionalization of the wafers with 3-aminopropyldimethylethoxysilane (97\%, Sigma Aldrich) was achieved by vapor phase silanization under reduced pressure (10 mbar, $24 \mathrm{~h}$ ) followed by rinsing with EtOH (VWR) to remove excess molecules.

Prior to the multilayer coating the substrates were immersed in PEI solution for $30 \mathrm{~min}$ to deposit an adhesion promoting layer. The build-up of (PSS/PDA $)_{5}$ and (PSS/PDA) $)_{5.5}$ multilayers by spray coating followed the procedure reported in Ref. [5]. 
Multilayers terminated with PSS were charged patterned by microcontact printing of PDA-TRITC following established protocols [49,50]. For this purpose we used polydimethylsiloxane (PDMS) stamps cast from lithographically patterned silicon masters.

On homogeneous substrates SPB were deposited by immersion in suspensions of varying ionic strength $(0.1 \mathrm{mM}$ to $1 \mathrm{M}, 0.1 \mathrm{wt} \%$ particles $)$ for $60 \mathrm{~min}$. Deposition of SPB on charge patterned surfaces was facilitated by drop casting in a humidified desiccator. The suspensions contained $0.1 \mathrm{wt} \%$ particles and $\mathrm{NaCl}$ concentrations of $1 \mathrm{mM}$ and $100 \mathrm{mM}$ respectively and were left on the substrates for at least $24 \mathrm{~h}$. Before drying, the non-adsorbed particles were removed by thorough washing. In-situ AFM imaging of wet samples showed that the amount of adsorbed particles did not change during this step.

\subsection{AFM imaging}

Imaging of the SPB assemblies was performed with a Nanowizard I AFM (JPK Instruments) operating in the Intermittent Contact Mode. We used soft cantilevers $(0.15 \mathrm{~N} / \mathrm{m}, 12 \mathrm{kHz}, \mathrm{CSC}-17$, MikroMasch Estonia) for in-situ imaging and stiff cantilevers (42 N/m, $300 \mathrm{kHz}$, OMCL-AC160TS-W2, Olympus) for imaging in air. To estimate the surface coverage in dependence on the ionic strength $100 \mu^{2}$ scans were recorded on at least three positions per sample using a Dimension IIIa AFM (Bruker). The particle density was determined by the automated counting procedure implemented in ImageJ.

\subsection{Force spectroscopy}

The colloidal particles were attached to calibrated, tipless AFM cantilevers (NSC12, Mikromasch) using a commercial epoxy glue (UHU Endfest 300) and a micromanipulator. The force constants as detected by the thermal noise method [37] ranged from $0.25 \mathrm{~N} / \mathrm{m}$ to $0.7 \mathrm{~N} / \mathrm{m}$. Force-distance curves were recorded in liquid using a Nanowizard I AFM. All solutions were adjusted to $\mathrm{pH} 4$ with $\mathrm{HCl}$ (Grüssing). Salt concentrations of $1 \mathrm{mM}$ and $100 \mathrm{mM}$ were obtained by addition of $\mathrm{NaCl}$.

\subsection{Electrophoretic mobility measurements}

Double-layer potential measurements of the microparticles were performed with a ZETAVIEW laser scattering video microscope (Particle Metrix GmbH). From the electrophoretic mobilities determined by the implemented image analysis algorithm, zeta potential values were calculated using the Smoluchowski equation [51]. All samples were strongly diluted in $1 \mathrm{mM} \mathrm{NaCl}$ solution. Their $\mathrm{pH}$ was adjusted by addition of $\mathrm{HCl}$.

\subsection{Field emission scanning electron microscopy (FESEM)}

FESEM specimen were prepared by drying one drop of a highly diluted suspension on a clean silicon wafer (CrysTec) at room temperature and coating with a platinum layer 
of $2 \mathrm{~nm}$ thickness using a sputter coater (Cressington 208HR) to make the specimen conductive. Micrographs were recorded on a LEO Gemini microscope (Zeiss) equipped with a field emission cathode operating at $3-5 \mathrm{kV}$, which corresponds to a lateral resolution of $2 \mathrm{~nm}$.

\subsection{Cryogenic transmission electron microscopy (cryo-TEM)}

Cryo-TEM sample were contrast enhanced in accordance to Ref. [52] by counterion exchange with $\mathrm{CsCl}$ and subsequent adsorption of bovine serum albumin (BSA). The SPB suspension was spread on a hydrophilized lacey carbon TEM grid (mesh size 200, Plano $\mathrm{GmbH}$ ) and vitrified with liquid ethane. Imaging was performed with a Zeiss EM922 OMEGA EFTEM (Zeiss NTS GmbH) at a temperature of $90 \mathrm{~K}$ and an acceleration voltage of $200 \mathrm{kV}$.

\subsection{Differential centrifugal sedimentation (DCS)}

The average size of the microparticles and their size distribution were measured using an analytical disc centrifuge (CPS Instruments CPS-24000) [53]. Within a hollow disc rotating at $5050 \mathrm{rpm}$ a gradient was prepared by layering eight sucrose solutions of decreasing density ( 8 to $3 \mathrm{wt} \%$ ) upon one another. $0.1 \mathrm{ml}$ of a dilute suspension of microparticles was placed on top of the gradient. The distribution of the microparticles was obtained by measuring the time required for the different species to reach a known position within the gradient. The concentration at this position and time was measured by light absorption at $405 \mathrm{~nm}$.

\section{Results and discussion}

Analysis of the adsorption behavior is an established methodology for investigating the interaction between particles and solid interfaces. Both the adsorption kinetics and the final surface coverage provide insights in this respect. In a recent study we found that SPB adsorption kinetics is diffusion-limited in the low surface coverage regime. After extended adsorption times the process runs into a jamming limit which always results in the formation of a SPB monolayer [36].

The saturation coverage of adsorbed SPB depends on both the surface charge and the ionic strength in solution. Figure 1B shows layers formed by the adsorption of particles carrying a PSS brush onto PDA and PSS terminated multilayers. While the SPB adsorb exclusively on the oppositely charged surface at low ionic strength, selectivity vanishes at high concentrations of added salt. The AFM images were recorded in-situ directly after excess particles had been removed by gentle washing. In the absence of attractive capillary forces a liquid-like ordering is preserved, indicating a random sequential adsorption of SPB. Obviously, the characteristic interparticle distance is strongly reduced by the addition of salt, which induces screening of the repulsive electrosteric interaction between the SPB. In Fig. 1C the saturation surface coverage on both oppositely and like-charged substrates is plotted as a function of the ionic strength. On PDA the surface coverage increases monotonically with the ionic strength, whereas 


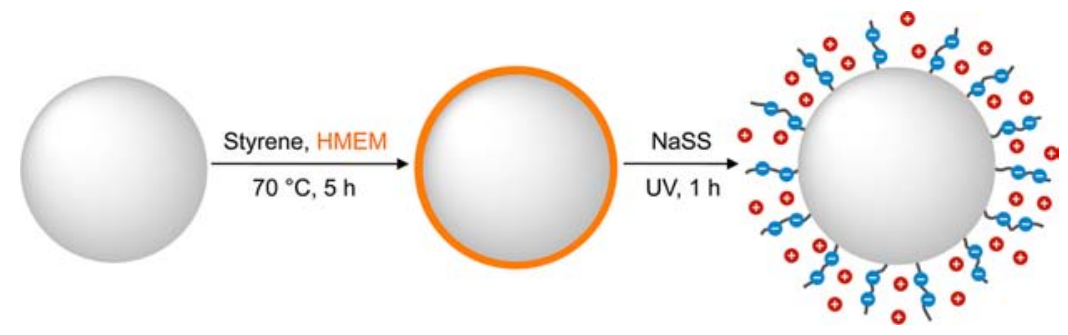

Fig. 2. Synthesis of micrometer-sized SPB: Microparticles bearing photocleavable groups at their surface were prepared by seeded growth polymerization of styrene and the polymerizable photoinitiator HMEM onto narrowly dispersed polystyrene seeds. Sodium styrene sulfonate (NaSS) was added and the suspension was irradiated by UV light. Covalently bound surface radicals thus generated initiated the polymerization of the water-soluble monomer resulting in polyelectrolyte brushes attached to the microparticles.

virtually no adsorption is observed on PSS terminated substrates below $10 \mathrm{mM}$ of added salt. Even higher salt concentrations lead to a gradual loss of the substrate selectivity. Matching surface coverage on both oppositely and like-charged substrates is found at salt concentrations above $100 \mathrm{mM}$. Interestingly, this value corresponds to the transition from the osmotic brush to the salted brush regime, which is known to have also a pronounced influence on the binding of proteins to SPB [54,55].

It is reasonable to assume that electrosteric forces dominate the interaction between SPB and charged surfaces in the osmotic brush regime. At low ionic strength SPB attachment to oppositely charged substrates is entropically favored due to counterion release, whereas electrostatic and steric repulsion suppress adsorption onto like-charged surfaces. In the salted brush regime, the polyelectrolyte corona is strongly collapsed due to screening rendering electrosteric interactions insignificant. Instead, attractive forces such as van der Waals or hydrophobic interactions become dominant facilitating SPB adsorption independent of the substrate type.

To assess the forces governing the interaction of SPB with charged surfaces directly, we chose the colloidal probe technique, where single colloidal particles are attached to AFM cantilevers. Due to the well-defined geometry a normalization of the measured force data by the Derjaguin approximation is possible. In order to perform such experiments, it was fundamental to modify micrometer-sized particles of suitable dimensions to be used as colloidal probes by grafting polyelectrolyte brushes. For this purpose, we selected cross-linked polystyrene microparticles, which were supplied from Soken Chemical \& Engineering Co.. Measurements of the distribution of the particle diameters by differential centrifugal sedimentation showed that the particles are narrowly dispersed with an average diameter of $4.8 \mu \mathrm{m}$. Their polydispersity defined as the weight-averaged diameter divided by the number-averaged diameter is as low as 1.001. Moreover, scanning electron micrographs revealed that the particles exhibit a uniform spherical shape with a smooth surface, which was essential to obtain a well-defined core-shell morphology after grafting the polyelectrolyte brush.

As illustrated in Fig. 2 the surface modification was carried out in two steps, beginning with coating the microparticles with a thin layer of photoinitiator. In the second step, surface-bound radicals, formed upon irradiation with UV light, initiated 

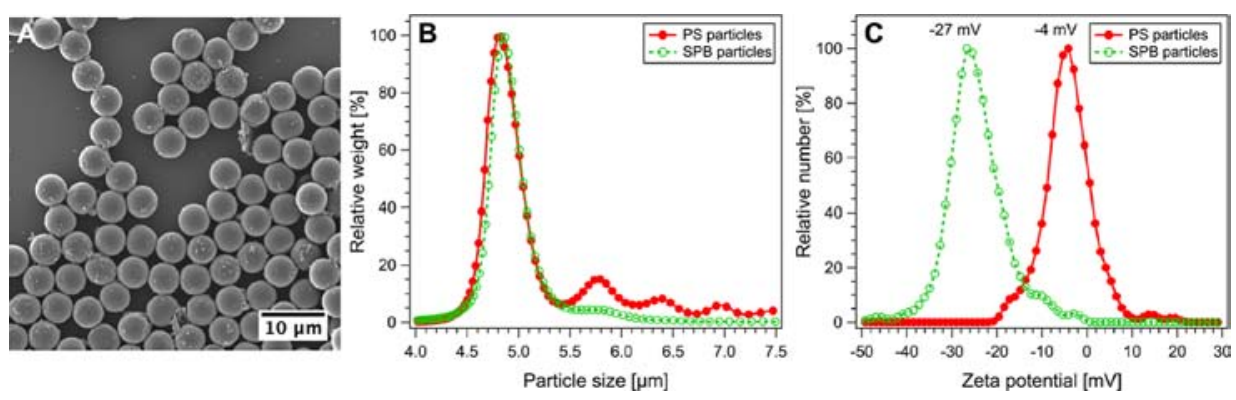

Fig. 3. Characterization of SPB microparticles. Size and shape of the microparticles are preserved during grafting of the brushes (A). In comparison to the PS seeds the brush decorated SPB microparticles show a significantly reduced amount of aggregates in differential centrifugal sedimentation measurements (B). The zeta potential at $\mathrm{pH} 4$ is clearly shifted to more negative values due to the grafted PSS chains (C).

the polymerization of the water-soluble monomer sodium styrene sulfonate resulting in covalently attached polyelectrolyte brushes. This method is well-established for the synthesis of SPB with submicron dimensions [24,56], but has to the best of our knowledge not been adapted to larger particles. While the general concept is similar to the one developed by Ballauff and co-workers for nanoparticles, special attention had to be paid to prevent aggregation of the micrometer-sized particles until the brush layer could add electrosteric stabilization.

Careful preparation was necessary to obtain homogeneous suspensions of the microparticles. For this purpose, the particles that were supplied as a freeze-dried powder were at first suspended in ethanol before an aliquot of water was added dropwise. Homogenization was accomplished by sonication. The photoinitiator layer was generated by swelling the cross-linked micrometer-sized seeds with a defined amount of styrene and then initiating the polymerization by addition of potassium persulfate. A water-soluble initiator was chosen to facilitate the polymerization at the surface of the particles. After a time given to form a "fresh" layer of polystyrene, a polymerizable photoiniator was added under starved conditions resulting in a copolymer shell on the seeds. It has to be noted that the microparticles kept their uniform spherical shape during the polymerization as corroborated by electron micrographs (Fig. 3A). This finding is not trivial, because given appropriate experimental conditions seeded growth polymerization from cross-linked particles can be used to prepare particles that exhibit defined anisotropic shapes, e.g. dumbbell-shaped particles [57,58]. In the final step, the water soluble monomer sodium styrene sulfonate was added and the suspension of the modified microparticles bearing covalently attached photoinitiator moieties at their surface was irradiated by UV light in a closed reaction chamber. As shown in earlier studies, multiple elastic scattering of the UV light within the turbid suspension enables the decomposition of the surface-bound photoinitiator. These radicals initiate the polymerization of the water-soluble monomer resulting in polyelectrolyte brushes covalently attached to the particles [24,56]. The decomposition of the applied photoinitiator results both in surface-bound radicals and free radicals in solution. The free polyelectrolyte chains in solution thus formed were removed by ultrafiltration against water. 
Manifold analytical techniques such as small-angle X-ray scattering [59], dynamic light scattering [24], cryogenic transmission electron microscopy [52] and electrophoretic measurements [60] have been used to comprehensively investigate the coreshell character of submicrometer-sized SPB. While such techniques are well-suited to study submicrometer-sized particles, they can be hardly applied on microscale objects. In order to estimate the brush thickness, a reference experiment with PS seeds of $169 \mathrm{~nm}$ in diameter was carried out in parallel to the photopolymerization onto the microscale seeds in the UV chamber. A brush thickness of $30 \mathrm{~nm}$ was obtained by DLS measurements of the hydrodynamic radii before and after the photopolymerization. As a first indication that a brush layer also formed on the microparticles may serve their significantly enhanced stability when dispersed in water. This was already evident from visual inspection. While the unmodified core particles showed rapid sedimentation in water and had thus to be kept in water/ethanol mixtures during surface modification, sedimentation of the brush coated microparticles proceeded much slower. This observation was quantitatively corroborated when measuring the size distribution of the particles by DCS (Fig. 3B). Considerable amounts of particle dimers, trimers and tetramers were found in freshly prepared aqueous suspensions of the PS seeds, which is in accord with their high sedimentation rate. Only a small portion of dimer ensembles are found after modification of the microparticles indicating the stabilization effected by the surrounding polyelectrolyte layer. This is further documented by optical measurements of the electrophoretic mobilities of individual microparticles before and after grafting the surface layer. The experiments were carried out at $\mathrm{pH} 4$ to exclude the influence of the carboxylic groups of the PS seeds on the mobility. Calculations of zeta potentials for hairy particles from electrophoretic mobilities are non-trivial [61]. In contrast to submicrometer-sized SPB, the influence of a $30 \mathrm{~nm}$ thick surface layer on the mobility of the $4.8 \mu \mathrm{m}$ SPB microparticles can be widely restricted to the effective charge of the particles. For this reasons, zeta potentials were calculated based on the Smoluchowski equation [51]. The low zeta potential of $-4 \mathrm{mV}$ of the PS seeds is in full accord with the modest stability of aqueous suspensions of these particles. There was a significant increase of the absolute value of the zeta potential $(-27 \mathrm{mV})$ after the photopolymerization again indicating the successful formation of the polyelectrolyte layer and the improved stability of the suspensions.

While the standard characterization methods all indicate a successful modification of the PS microparticles, they reflect the averaged properties of a large ensemble of particles. To investigate the single particles by CP-AFM we attached PS core particles and PSS modified particles to tipless cantilevers. In analogy to the zeta potential measurements all AFM experiments were conducted at $\mathrm{pH} 4$ to screen contributions stemming from the carboxyl moieties of the core particles. Figure 4A,B displays typical forcedistance curves obtained on amino-functionalized $\mathrm{Si}$ wafers, which carry a positive net charge under acidic conditions. Independent of the ionic strength the PS core particles show a hard repulsive interaction without noticeable adhesion upon retraction. Retract curves of the modified particles on the other hand display a strongly adhesive interaction most pronounced at low ionic strength. Despite the fact that SPB adsorption is promoted by the addition of salt, electrostatic screening results in significantly decreased binding strengths. Similar trends apply to PDA terminated polyelectrolyte multilayers as substrates with the qualitative difference that we often observe a less 

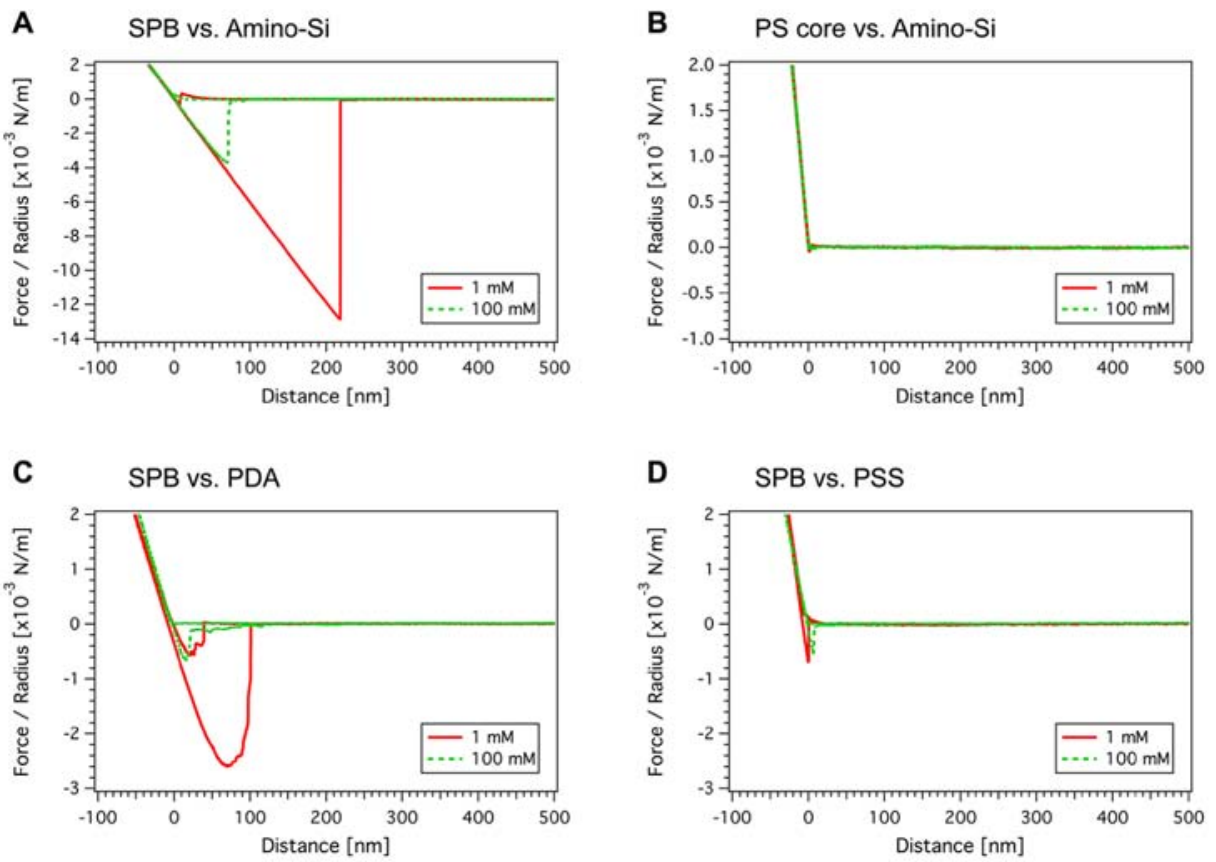

Fig. 4. Force curves of PS microparticles and SPB microparticles. While the SPB microparticle shows strong adhesion to the oppositely charged, amino-functionalized substrate (A), little adhesion is found for the PS reference particle (B). The strength of the attractive interaction can be controlled by the ionic strength and the charge of the substrate as shown for PDA (C) and PSS (D) terminated multilayers. All data was obtained at $\mathrm{pH} 4$.

rapid, stepwise detachment upon retraction of the cantilever (Fig. 4C). Measurements in the presence of PSS terminated multilayers (Fig. 4D) on the other hand displayed small forces of adhesion, which were independent of the ionic strength and can be attributed to secondary interactions and chain entanglements [62].

We have to note that only about half of the probed SPB microparticles showed significant differences from the bare PS cores indicating an incomplete conversion during photopolymerization. Also with an increasing number of measurements the force of adhesion decreased steadily indicating that PSS chains are partially torn out of the brush. Considering the charged surface as a multivalent counterion very strong binding is expected [35]. In our measurements the retract speeds $(500 \mathrm{~nm} / \mathrm{s})$ were rather high possibly preventing a stepwise detachment of single charged polymer groups. Whereas more quantitative investigations will have to take these aspects into account, we were primarily interested in a qualitative assessment of the strength of SPB adhesion at high and low ionic strengths.

Both the adsorption study and the single particle study demonstrated the pronounced substrate selectivity of SPB, which is a prerequisite for the construction of SPB surface assemblies on charge patterned substrates. To create substrates with welldefined positive and negative regions we printed labeled PDA-TRITC onto PSS termi- 

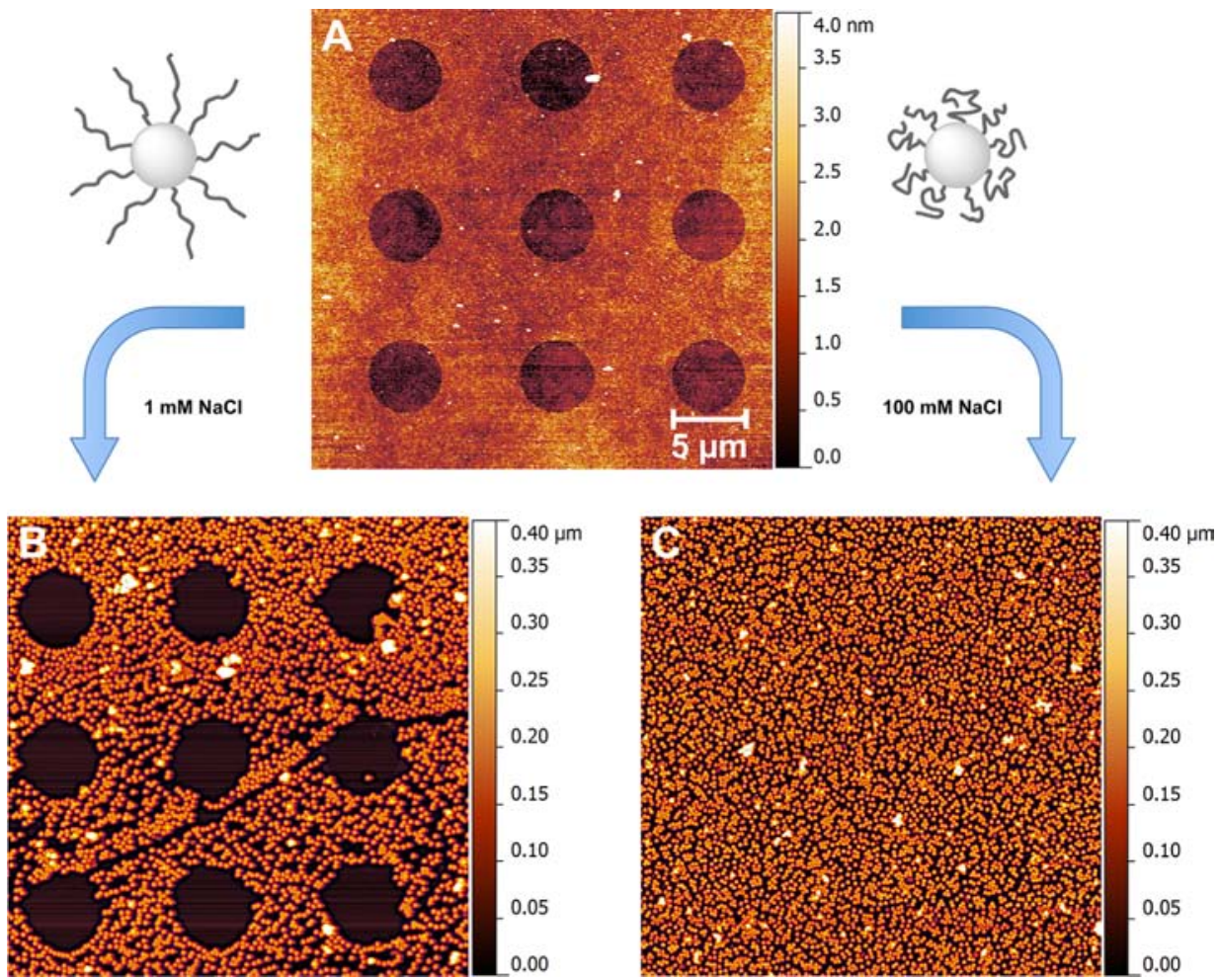

Fig. 5. SPB adsorption onto charge patterned substrates. The AFM image shows negatively charged areas as dark circles surrounded by a positively charged matrix (A). At low ionic strength the particles adsorb selectively onto the oppositely charged regions (B). Substrate selectivity is completely suppressed at high concentrations of added salt (C).

nated multilayers using hydrophilized PDMS stamps. Successful pattern reproduction was proven by fluorescence microscopy and AFM imaging. A typical microcontact print consisting of negative circles and a surrounding positive matrix is displayed in Fig. 5A. Onto the patterned region we applied droplets of SPB suspensions containing $1 \mathrm{mM}$ and $100 \mathrm{mM}$ of $\mathrm{NaCl}$. These samples were stored in a humidified desiccator for at least $24 \mathrm{~h}$ before washing with water and drying in a nitrogen stream. As shown in Fig. 5B the SPB adsorbed only onto oppositely charged areas at low ionic strength leading to excellent pattern reproduction. Increasing the ionic strength to $100 \mathrm{mM}$ on the other hand resulted in a complete loss of substrate selectivity (Fig. 5C). As confirmed by fluorescence microscopy the pattern fidelity was unaffected by the deposition process. The observed non-selectivity can therefore be attributed to attractive secondary interactions, such as van der Waals forces or hydrophobic interaction, dominating the $\mathrm{SPB}$ adsorption in the salted brush regime.

As further demonstrated in Fig. 6, charge patterning is a powerful tool for controlling surface order as well as the patterning of sub-micrometer SPB on the micrometer scale and even up to macroscopic dimensions. 

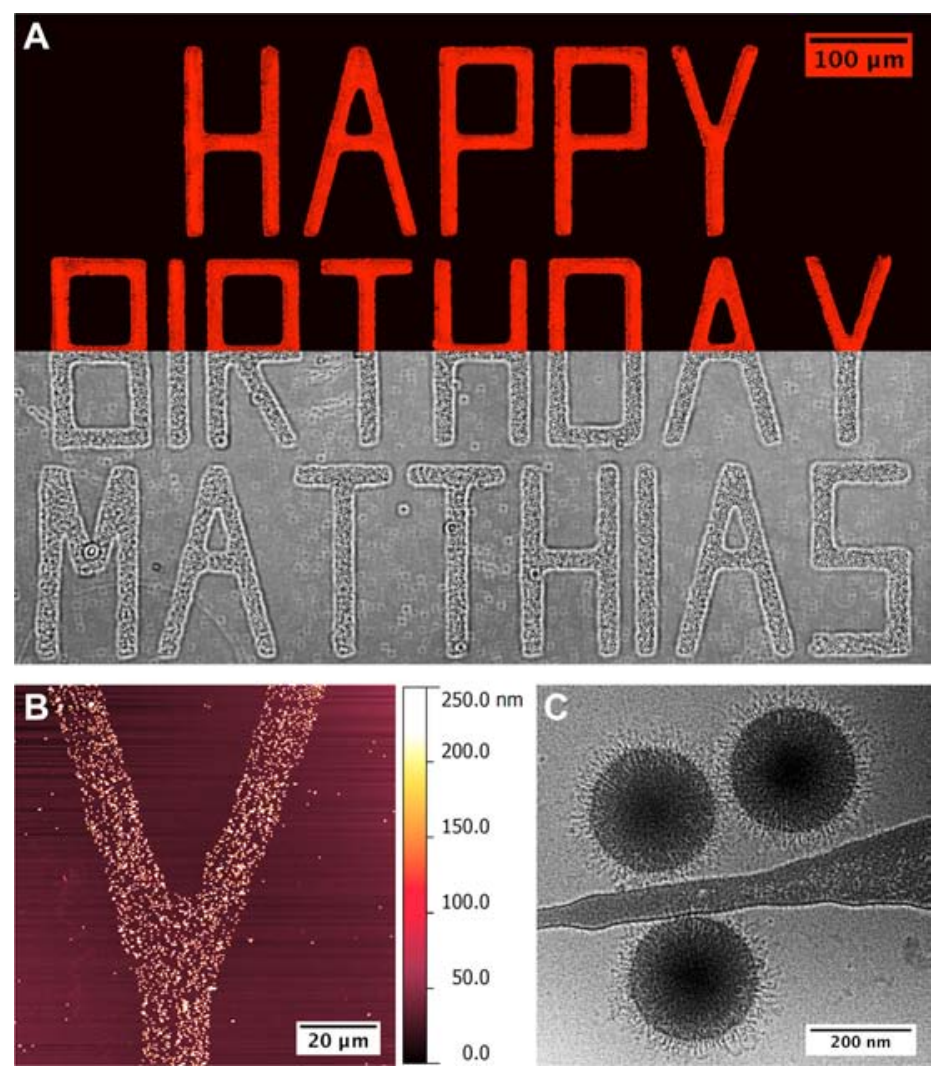

Fig. 6. Hierarchical structuring by selective SPB adsorption: Millimeter-sized patterned areas were produced by microcontact printing with a custom PDMS stamp (top: fluorescence image, bottom: phase contrast micrograph after SPB deposition) (A). Micron-sized particle arrays are formed upon the adsorption of SPB (AFM) (B). The Cryo-TEM image of individual SPB contrasted by the adsorption of bovine serum albumin demonstrates the core-shell architecture $(\mathrm{C})$.

\section{Conclusions}

We investigated the interaction of spherical polyelectrolyte brushes with charged surfaces. Particle monolayers prepared by SPB adsorption onto positively and negatively charged polyelectrolyte multilayers showed a distinct dependence of the surface coverage on the concentration of added $\mathrm{NaCl}$. The particles adsorbed exclusively onto oppositely charged surfaces at ionic strengths up to $10 \mathrm{mM}$, whereas higher salt concentrations resulted in a gradual loss of substrate selectivity. While counterion release and electrosteric repulsion govern the interaction between SPB and charged surfaces in the osmotic brush regime, attractive secondary interactions become dominant in the salted brush regime.

The strength of the interaction forces can be assessed on a single particle level by colloidal probe AFM. For this purpose we equipped cross-linked polystyrene mi- 
croparticles with UV sensitive moieties and grafted chains of polystyrene sulfonate from the particle surfaces. This modification lead to significantly enhanced colloidal stability in water. Further, the PSS grafted microparticles displayed a strongly negative zeta potential even under acidic conditions, where the bare core particles carrying only carboxyl groups are mostly neutral. In analogous single particle AFM measurements the presence of grafted PSS chains resulted in a massively altered interaction with positively charged surfaces. Whereas the bare cores show no attraction towards the substrate under acidic conditions, the modified particles adhere strongly. The force of adhesion can be regulated via the ionic strength in the surrounding medium.

Further, we used charge patterned substrates produced by polymer-on-polymer stamping to arrange SPB into patterned arrays. As expected from the experiments with homogenous surfaces site-selective SPB attachment occurred only at low ionic strength. While this has been demonstrated with hard particles [45], the stimuli-responsive nature of the brush layer avoids an irreversible hit-stick behavior and could therefore allow us to remove structural defects by annealing. Considering the numerous chemical modifications which have been reported for SPB [29,33,63], our study opens exiting avenues for the production of stimuli-responsive materials with a hierarchical internal organization.

Figure 6 shows a particular example of such a hierarchical structure containing two levels of hierarchy: the internal core-shell character of the particles and their positional order on the printed micropattern. As one can clearly see, completely novel functionalities arise, like the use of nanoparticles for communication, which brings us to the main aim of this manuscript:

Lieber Matthias, wir wünschen Dir alles Gute zu Deinem Geburtstag! Es ist uns eine Freude und ein Privileg mit diesem Manuskript einen wissenschaftlichen Beitrag zu Deiner Geburtstagsfeier leisten zu können! Herzliche Gratulation!

\section{Acknowledgement}

This work has been conducted within the special collaborative research project SFB 840 (project B5) and was funded by the German Science Foundation (DFG). The authors would like to acknowledge Carmen Kunert and Dr. Simone Wagner for FESEM imaging as well as Dr. Markus Drechsler for cryo-TEM imaging. We also thank Nicolas Helfricht for his assistance with the colloidal probe preparations.

\section{References}

1. G. Decher, Science 277 (1997) 1232.

2. G. Decher, J. D. Hong, and J. Schmitt, Thin Solid Films 210 (1992) 831.

3. S. T. Dubas and J. B. Schlenoff, Macromolecules 32 (1999) 8153.

4. D. Yoo, S. S. Shiratori, and M. F. Rubner, Macromolecules 31 (1998) 4309.

5. A. Izquierdo, S. S. Ono, J.-C. Voegel, P. Schaaf, and G. Decher, Langmuir 21 (2005) 7558.

6. C. H. Porcel, A. Izquierdo, V. Ball, G. Decher, J.-C. Voegel, and P. Schaaf, Langmuir 21 (2005) 800.

7. J. B. Schlenoff, S. T. Dubas, and T. Farhat, Langmuir 16 (2000) 9968. 
8. J. E. Wong, H. Zastrow, W. Jaeger, and R. von Klitzing, Langmuir 25 (2009) 14061.

9. S. Schmidt, H. Motschmann, T. Hellweg, and R. von Klitzing, Polymer 49 (2008) 749.

10. S. Schmidt, M. Zeiser, T. Hellweg, C. Duschl, A. Fery, and H. Möhwald, Adv. Funct. Mater. 20 (2010) 3235.

11. J. Gensel, T. Borke, N. Pazos-Pérez, A. Fery, D. V. Andreeva, E. Betthausen, A. H. E. Müller, H. Möhwald, and E. V. Skorb, Adv. Mater. 24 (2012) 985.

12. O. D. Velev and S. Gupta, Adv. Mater. 21 (2009) 1897.

13. Y. Xia, B. Gates, Y. Yin, and Y. Lu, Adv. Mater. 12 (2000) 693.

14. W. S. Tan, Z. Zhu, S. A. Sukhishvili, M. F. Rubner, and R. E. Cohen, Macromolecules 44 (2011) 7767.

15. C. H. Lu, H. Möhwald, and A. Fery, Soft Matter 3 (2007) 1530.

16. A. Schweikart, N. Pazos-Pérez, R. A. Alvarez-Puebla, and A. Fery, Soft Matter 7 (2011) 4093.

17. S. T. Milner, Science 251 (1991) 905.

18. M. Ballauff and O. Borisov, Curr. Opin. Colloid Interface Sci. 11 (2006) 316.

19. M. A. C. Stuart, W. T. S. Huck, J. Genzer, M. Müller, C. Ober, M. Stamm, G. B. Sukhorukov, I. Szleifer, V. V. Tsukruk, M. Urban, F. Winnik, S. Zauscher, I. Luzinov, and S. Minko, Nat. Mater. 9 (2010) 101.

20. M. Biesalski, D. Johannsmann, and J. Rühe, J. Chem. Phys. 117 (2002) 4988.

21. T. Chen, R. Ferris, J. M. Zhang, R. Ducker, and S. Zauscher, Prog. Polym. Sci. 35 (2010) 94.

22. G. Lagaly (Ed.), Molecular Organisation on Interfaces, Springer Berlin/Heidelberg (2002), p. 34.

23. A. Jusufi, C. N. Likos, and M. Ballauff, Colloid Polym. Sci. 282 (2004) 910.

24. X. Guo and M. Ballauff, Langmuir 16 (2000) 8719.

25. X. Guo and M. Ballauff, Phys. Rev. E 64 (2001) 051406.

26. Y. Mei, K. Lauterbach, M. Hoffmann, O. V. Borisov, M. Ballauff, and A. Jusufi, Phys. Rev. Lett. 97 (2006) 158301.

27. G. Dominguez-Espinosa, A. Synytska, A. Drechsler, C. Gutsche, K. Kegler, P. Uhlmann, M. Stamm, and F. Kremer, Polymer 49 (2008) 4802.

28. C. Gutsche, M. M. Elmahdy, K. Kegler, I. Semenov, T. Stangner, O. Otto, O. Ueberschär, U. F. Keyser, M. Krueger, M. Rauscher, R. Weeber, J. Harting, Y. W. Kim, V. Lobaskin, R. R. Netz, and F. Kremer, J. Phys.-Condens. Matter 23 (2011) 17.

29. A. N. Korovin, V. G. Sergeyev, O. A. Pyshkina, C. Hanske, A. Fery, A. Wittemann, and L. Tsarkova, Macromol. Rapid Commun. 32 (2011) 462.

30. M. Schrinner, M. Ballauff, Y. Talmon, Y. Kauffmann, J. Thun, M. Möller, and J. Breu, Science 323 (2009) 617.

31. A. Wittemann and M. Ballauff, Phys. Chem. Chem. Phys. 8 (2006) 5269.

32. F. Schacher, E. Betthausen, A. Walther, H. Schmalz, D. V. Pergushov, and A. H. E. Müller, AC. Nano 3 (2009) 2095.

33. Y. Lu, A. Wittemann, and M. Ballauff, Macromol. Rapid Commun. 30 (2009) 806.

34. H. Gliemann, Y. Mei, M. Ballauff, and T. Schimmel, Langmuir 22 (2006) 7254.

35. Y. Mei, A. Wittemann, G. Sharma, M. Ballauff, T. Koch, H. Gliemann, J. Horbach, and T. Schimmel, Macromolecules 36 (2003) 3452.

36. C. Hanske, C. Schneider, M. Drechsler, A. Wittemann, and A. Fery, Phys. Chem. Chem. Phys. 14 (2012) 4196.

37. H.-J. Butt, B. Cappella, and M. Kappl, Surf. Sci. Rep. 59 (2005) 1.

38. H.-J. Butt, Biophys. J. 60 (1991) 1438.

39. W. A. Ducker, T. J. Senden, and R. M. Pashley, Nature 353 (1991) 239.

40. E. Spruijt, M. A. Cohen Stuart, and J. van der Gucht, Macromolecules 43 (2010) 1543.

41. J. Bünsow, J. Erath, P. M. Biesheuvel, A. Fery, and W. T. S. Huck, Angew. Chem.-Int. Ed. 50 (2011) 9629.

42. S. Block and C. A. Helm, Macromolecules 42 (2009) 6733.

43. V. Bosio, F. Dubreuil, G. Bogdanovic, and A. Fery, Colloid Surf. A-Physicochem. Eng. Asp. 243 (2004) 147.

44. Q. Chen, G. W. de Groot, H. Schönherr, and G. J. Vancso, Eur. Polym. J. 47 (2011) 130.

45. H. Zheng, M. F. Rubner, and P. T. Hammond, Langmuir 18 (2002) 4505. 
46. T. Chen, R. Jordan, and S. Zauscher, Small 7 (2011) 2148.

47. M. Hoffmann, A. Jusufi, C. Schneider, and M. Ballauff, J. Colloid Interface Sci. 338 (2009) 566.

48. W. Kern and D. A. Puotinen, RC. Rev. 31 (1970) 187.

49. X. P. Jiang, H. P. Zheng, S. Gourdin, and P. T. Hammond, Langmuir 18 (2002) 2607.

50. M. Pretzl, A. Schweikart, C. Hanske, A. Chiche, U. Zettl, A. Horn, A. Böker, and A. Fery, Langmuir 24 (2008) 12748.

51. M. von Smoluchowski, Z. Phys. Chem. 92 (1917) 129.

52. A. Wittemann, M. Drechsler, Y. Talmon, and M. Ballauff, J. Am. Chem. Soc. 127 (2005) 9688.

53. D. J. Scott, S. E. Harding, and A. J. Rowe (Eds.), Analytical Ultracentrifugation: Techniques and Methods, The Royal Society of Chemistry, Cambridge (2005), p. 270.

54. A. Wittemann, B. Haupt, and M. Ballauff, Phys. Chem. Chem. Phys. 5 (2003) 1671.

55. A. Wittemann, B. Haupt, and M. Ballauff, Z. Phys. Chem.-Int. J. Res. Phys. Chem. Chem. Phys. 221 (2007) 113.

56. M. Schrinner, B. Haupt, and A. Wittemann, Chem. Eng. J. 144 (2008) 138.

57. J.-W. Kim, R. J. Larsen, and D. A. Weitz, Adv. Mater. 19 (2007) 2005.

58. E. B. Mock, H. D. Bruyn, B. S. Hawkett, R. G. Gilbert, and C. F. Zukoski, Langmuir 22 (2006) 4037.

59. M. Ballauff, Prog. Polym. Sci. 32 (2007) 1135.

60. M. L. Jiménez, A. V. Delgado, S. Ahualli, M. Hoffmann, A. Witteman, and M. Ballauff, Soft Matter 7 (2011) 3758.

61. H. Ohshima and K. Makino, Colloids Surf. A 109 (1996) 71.

62. R. Pericet-Camara, G. Papastavrou, S. H. Behrens, C. A. Helm, and M. Borkovec, J. Colloid Interface Sci. 296 (2006) 496.

63. Y. Lu, Y. Mei, R. Walker, M. Ballauff, and M. Drechsler, Polymer 47 (2006) 4985. 